\title{
Spatial correlation of gene expression measures in tissue microarray core analysis
}

\author{
MATHIEU EMILY*†, DIDIER MOREL $\ddagger$, RAPHAEL MARCELPOIL $\ddagger$ and OLIVIER FRANÇOIS $\dagger$ \\ $\dagger$ TIMC-TIMB, Faculty of Medicine, 38706 La Tronche cedex, France \\ $\ddagger$ Tripath Imaging Europe, 29 Boulevard des Alpes, 38246 Meylan, France \\ (Received 7 May 2004; in final form 21 December 2004)
}

\begin{abstract}
Tissue microarrays (TMAs) make possible the screening of hundreds of different tumour samples for the expression of a specific protein. Automatic features extraction procedures lead to a series of covariates corresponding to the averaged stained scores. In this article, we model the random geometry of TMA cores using voronoi tesselations. This formalism enables the computation of indices of spatial correlation of stained scores using both classical and novel approaches. The potential of these spatial statistics to correctly discriminate between diseased and non-diseased cases is evaluated through the analysis of a TMA containing samples of breast carcinoma data. The results indicate a significant improvement in the breast cancer prognosis.
\end{abstract}

Keywords: Tissue microarrays; Random geometry; Spatially marked point process; Voronoi tesselation; Cancer prognosis

\section{Introduction}

Tissue microarrays (TMAs) consist of high-throughput tissue-based tools that allow for the immunohistological analysis of a large sample of tumour tissues [1,2]. TMAs enable the screening of hundreds of different tumour samples for the expression of a specific protein. They are used in in situ gene marker validation and discovery in cancer research projects thanks to their ability to rapidly evaluate the role of normal and abnormal genes and gene products from numerous tissues at once, and ultimately correlate genotypes with phenotypes. A typical example of a tissue array application is in searching for oncogenes amplifications in vast tumour tissue panels, encompassing differing stages and grades of disease.

Many automated treatments of TMAs are available, allowing measurements of fluorescence intensity, probe colocalization and other measures of protein expression. Although efficient software tools for counting cells and objects and estimating morphometric parameters exist, solutions for the statistical analysis of staining results are still lacking. A major difficulty is that TMAs core image information cannot be summarized easily and the relationships between genetic expression and tissue structure are still poorly understood.
In providing supervised classifiers that can assist pathologists in the prognosis of a disease, a crucial question is to know which covariates and indices provide the most information. In this paper, we evaluate the potential of spatial statistical quantities within the mathematical formalism of marked point processes [3] to correctly discriminate between diseased and non-diseased cases. Our approach to the statistical description of TMAs' cores assumes pre-analysed images where cell parameters have been segmented. Given these data, two types of statistical indices can be computed using the Voronoi tesselation associated with the stained cells. The first indices correspond to the classical spatial correlation indices of Moran [4] and Geary [5] (see also [6]). The second indices are modified versions which attempt to account for the correlation of levels of expression in the highly expressed genes.

This article is organized as follows: In section 2, we give a short account of TMA technology, and the existing automatic treatments of such data; in section 3, we describe statistical summaries of the tissues based on random geometry concepts; in section 4, the approach is illustrated by the analysis of a TMA containing samples of breast carcinoma data and tests based on the spatial statistics are assessed from this dataset.

*Corresponding author. E-mail: mathieu.emily@imag.fr 


\section{TMAs}

\subsection{Overview}

TMAs are a method of re-locating tissue from conventional histologic paraffin blocks in order to see tissues from multiple patients or blocks on the same slide. Hundreds of tiny cylindrical tissue cores (typically $0.6 \mathrm{~mm}$ diameter) are densely and precisely arrayed into a single histologic paraffin block. The block may be divided into serial 4-8 $\mu \mathrm{m}$ thick sections, which we refer to as tissue array slides. Typically, cores contain small histologic sections from unique tissues or tumours. These tissue array slides serve as targets for immunohistochemical staining reactions.

Several biomarkers can be embedded in TMAs. For example, the cell proliferation biomarker Ki-67, has been shown to be significantly associated with survival in prostate cancer [7], nuclear phospho-beta-catenin in colorectal cancer is associated with a better prognosis [8], maspin is associated with breast cancer [9] and has a reverse correlation with mutant p53 [10], estrogen receptor and progesterone are utilized as well [11], etc.

Each tissue array slide yields information about protein staining pattern, distribution, intensity, background and target tissue. In many cases, each spot on a TMA is scored by a skilled pathologist and recorded manually. High-resolution digital images are automatically assembled into montages and usually saved as a TIFF format. Platforms that help organize TMA datasets and provide elementary analysis tools are available on the internet at University of California, Los Angeles (TMATRIX, http://www.genetics.ucla.edu/tissuearray), Johns Hopkins University (TMAJ Software, http://www. tmaj.com) or Stanford University (http://genome-www. stanford.edu/TMA/).

Several computer platforms (most of them commercial) enable the analysis of raw TMA images. These digital images are used for analysis of both anatomical features and fine tissue structures. Machine learning algorithms combined with data mining allow the extraction of essential features that classify particular tissue elements: geometric features such as the locations of cells, area, perimeter, compactness, elongation, and transmittance. These parameters may describe the shape and orientation of cells, nuclei, cytoplasms and membranes. The optical densities of pixels within each cell are recorded as well, and summarized from standard statistics (mean, median, standard deviation, quartiles). Cells can be in different states such as in differentiation, mitosis and apoptosis. As pointed out by Liu et al. [12], the extraction of such features usually leads to highly correlated covariates.

\subsection{Data description}

A clinical study was designed to identify a set of proteins for which over-expression has diagnostic significance in breast cancer and can be assessed by immunohistochemical assays and quantitative bright field microscopy for routine diagnostic of breast cancer. The study was based on a cohort of 132 patients including good and bad outcome patients. Good outcome are those that are disease free after five years, bad outcome is defined as recurrence or death within five years. The subset used in the article consists of 31 patients' data taken from this clinical study.

TMAs were made of 60 cores each, organized following a grid with 6 columns and 10 rows called TMA key. Good and bad outcome patients were represented twice. Additional cores from normal breast tissue, liver and tonsil tissues were used as staining controls and as helpers in the placement of the cores. For the purpose of this methodological study the marker investigated was a 32$\mathrm{kDa}$ dopamine and cyclic adenosine $3^{\prime}, 5^{\prime}$-monophosateregulated phosphoprotein named DARPP-32. A recent study has showed that both DARPP-32 and t-DARPP mRNAs were frequently over-expressed in carcinomas of the breast, prostate, colon and stomach compared with normal tissue samples [13].

The immunohistochemical assay was optimized for staining in a series of experiments varying the antibody concentration and the antigen retrieval methods (data not reported). The staining was optimized to have as low as possible staining in the good outcome and still a high staining in the bad outcome patients. Every TMA was processed with both a counterstain (Hematoxylin) and an immunochemical assay including polyclonal antibodies targeting the DARPP-32 protein revealed by DAB (brown). At the time of the study, these polyclonal antibodies had not yet been sorted and purified and therefore, the biological meaning of the staining intensity must be considered as preliminary data.

TMAs were scanned at $20 \times$ by the Tripath Imaging, Inc., proprietary slide scanning systems. Cores images were processed with Tripath Imaging, Inc., proprietary imaging system, using chromogen separation techniques to best separate marker contribution (DAB) from counterstain intensity (Hematoxylin). Each core was automatically segmented to obtain the position of every cell and finally extract features at cell and core level. These features were exported to a file used for further analysis.

\section{Statistical modeling}

\subsection{Tissues and tesselations}

Statistics in the area of TMAs have focused on the specific point of evaluating the predictive power of classification methods based on a set of covariates obtained via the automated features extraction algorithms. The package of Liu et al. [14] includes unsupervised analysis using hierarchical clustering. Liu et al. [12] present statistical methods for relating TMA data to censored time-to-event data (post-operative survival or time to first tumour recurrence). They review methods for 
evaluating the predictive power of Cox regression models and show how to test whether biomarker data contains predictive information above and beyond standard pathology covariates. They also used data mining methods for characterizing high risk patients with simple biomarker rules (survival trees, patient rule induction method).

The approach presented here provides a simple mathematical representation of a TMA core based on random tesselation. It starts from a subset of cell locations (a list of two dimensional coordinates) and reconstructs a statistical model of the tissues from which a number of tests can be performed. Tissues are reconstructed using the Voronoi tesselation. Modelling tissues with tesselations is a rather classical idea in biology and biometrics [15]. More specifically, let us recall a definition of the Voronoi tesselation. Consider a finite subset of locations or points in the plane. For each location $p$, the Voronoi cell $V(p)$ is the set of all points that are closer to $p$ than to all other locations in the subset.

The novelty of the approach presented here resides in the fact that stained scores are attached to the idealized Voronoi cells. Doing so, tissues can be viewed as wellstudied abstract objects called marked point processes $[3,16]$. The paradigm presented here is that the marked point process should contain a model of interactions between cells and markers that can be measured using the geometric information of the tesselation.

\subsection{Local correlation indices}

A challenge for TMA analysis is to pick a staining score (guided by prior biological knowledge) and find an index that could represent information at the level of the entire tissue. Thus our first concern is to define biomarker expression indices in relation to the spatial conformation of the tissues. Here the theory of marked point process comes to play. Let $X_{i}$ denote any score value associated with the cell $i$. For instance, $X_{i}$ can be the optical density of the biomarker estimated locally. Then, for a box $B$, let $n(B)$ be the number of cells in $B$ and $n_{i}(B)$ the number of neighbouring cells of $i$ in the Voronoi tesselation. The Moran's index can be defined as

$$
\rho_{\mathrm{M}}(B)=\frac{c(B)}{\sigma^{2}(B)}
$$

where $c(B)$ is defined by

$$
c(B)=\frac{1}{n(B)} \sum_{i} \frac{1}{n_{i}(B)} \sum_{j \sim i}\left(X_{i}-\bar{X}\right)\left(X_{j}-\bar{X}\right),
$$

$j \sim i$ means that $i$ and $j$ are neighbors, $\bar{X}$ is the mean value of $X$ and $\sigma^{2}(B)$ the unbiased variance of $X$ in $B$.
Similarly, the Geary's index is

$$
\rho_{\mathrm{G}}(B)=\frac{d(B)}{\sigma^{2}(B)}
$$

where

$$
d(B)=\frac{1}{2 n(B)} \sum_{i} \frac{1}{n_{i}(B)} \sum_{j \sim i}\left(X_{i}-X_{j}\right)^{2}
$$

Both indices are closely related to the notion of spatial autocorrelation of scores. In equation $(1), c(B)$ is equal to the local spatial covariance of scores in $B$, whereas in equation (2), $d(B)$ is equal to the local variance. Small values of the Moran index $\rho_{\mathrm{M}}(B)$ indicate that the spatial autocorrelation is weak. When the Moran index $\rho_{\mathrm{M}}(B)$ is close to 1 , the scores are strongly correlated and the Geary's index may be close to 0 . At the other extreme, when the Moran index $\rho_{\mathrm{M}}(B)$ is close to -1 , Geary's index may be high depending on the structure of the Voronoi tesselation. Apanasovich et al. [17] have recently applied Moran tests in order to detect the spatial correlation of specific morphologically changed structures that are known to be precursors of colon cancer development.

\subsection{New indices}

Moran's and Geary's indices may sometimes miss useful spatial information within a TMA core. For example, consider the two cores represented in figure 1. These two images are associated with two different outcomes of breast carcinoma (the first is good, the second bad). The stained scores (average local cell optical densities) are reported on the tesselations in grey color levels. This example reflects a weakness of the Moran's index and the Geary's index in discriminating the two clinical outcomes. In the first case, Moran's index is equal to $\rho_{\mathrm{M}}=0.68$ and Geary's index is equal to $\rho_{\mathrm{G}}=0.63$. The values do not differ significantly in the second case, where $\rho_{\mathrm{M}}=0.68$ and $\rho_{\mathrm{G}}=0.65$.

These examples emphasize the need for other indices. Clearly, the second case displays more contrasted scores than the first one and the presence of small clusters is not detected by the Moran and Geary indices. This suggests that introducing a threshold could improve the statistical prognosis of the outcome. Let $\theta$ be the threshold, and let $B_{\theta}$ denote the subset of cells in $B$ for which the scores are greater than $\theta$

$$
B_{\theta}=\left\{i \in B, X_{i}>\theta\right\}
$$

The thresholded indices are defined as follows:

$$
\rho_{\theta}(B)=\frac{c_{\theta}(B)}{\sigma_{\theta}(B) \sigma(B)}
$$



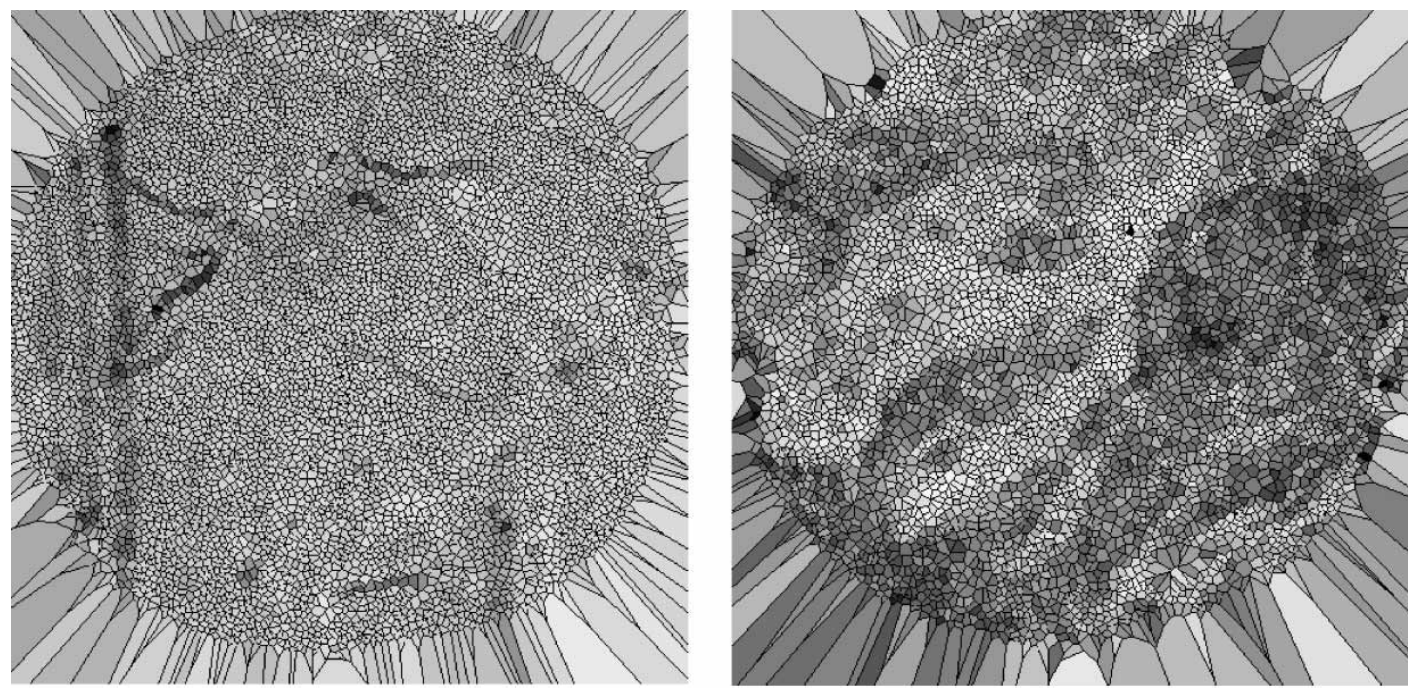

Figure 1. Voronoi tesselation models obtained from TMA cores of two cases: good outcome (left) and bad outcome (right). The stained scores (average local cell optical densities) are reported on the tesselations in grey color levels.

where

$$
c_{\theta}(B)=\frac{1}{n\left(B_{\theta}\right)} \sum_{i \in B_{\theta}} \frac{1}{n_{i}(B)} \sum_{j \sim i}\left(X_{i}-M_{\theta}\right)\left(X_{j}-\bar{X}\right)
$$

with $n\left(B_{\theta}\right)$ the number of cells in $B_{\theta}, M_{\theta}$ the average score over the neighbours of cells belonging to $B_{\theta}$ :

$$
M_{\theta}=\frac{1}{n\left(B_{\theta}\right)} \sum_{i \in B_{\theta}} \frac{1}{n_{i}(B)} \sum_{j \sim i} X_{j}
$$

and $\sigma_{\theta}(B)$ the square root of

$$
\sigma_{\theta}^{2}(B)=\frac{1}{n\left(B_{\theta}\right)} \sum_{i \in B_{\theta}} \frac{1}{n_{i}(B)} \sum_{j \sim i}\left(X_{i}-M_{\theta}\right)^{2} .
$$

The indices $\rho_{\theta}$ are obvious generalisations of the Moran's index. The value $M_{\theta}$ is of first order while $\rho_{\theta}$ is of second order. Before computing the index, the value of the threshold $\theta$ must be set. Assuming large threshold values, large values of $\rho_{\theta}$ should be observed if clusters of cells with over-expressed genes are observed. Improved classification performances over the classical indices may therefore be expected with respect to tissues with such properties. Negative values of $\rho_{\theta}$ could indicate that high scores are isolated events in the tissue. Values close to zero indicate weak correlations of high scores with those of their neighbours. As an illustration, the values $\rho_{\theta}$ for the two cores presented in figure 1 are equal to $\rho_{\theta}=$ 2.49 in the first case and 0.82 in the second case. In these examples, $\theta$ was taken equal to the median of scores. In general, $\theta$ can be chosen to optimize a classification procedure into pathologic and non-pathologic cases (see "Evaluation of the impact of geometry" section). Here the difference between the two cores becomes significant in comparison to the values of Moran and Geary indices.

\section{Evaluation of the impact of geometry}

\subsection{Classifiers}

This section assesses the discriminant power of the indices introduced in section 3.3 with the dataset presented in the second section. Each index defines a test for relating staining scores of biomarkers to clinical outcome information.

The ability of a test to discriminate diseased cases from normal cases can be evaluated using receiver operating characteristic (ROC) curve analyses [18]. Regarding threshold dependent methods such as $\rho_{\theta}$, the threshold was chosen in order to optimize the classification performances, i.e. the parameter associated with the maximal sensitivity and specificity.

Linear or non-linear discriminant analysis and flexible or logistic regression are utilized as well. In order to apply such analyses, there is a need to pool multiple spot measurements across each case (patient). Since TMAs case data contain pooled estimates of spot biomarker staining scores across each case, the simplest pooling methods are to form the mean, median, standard deviation of the spot measurements (standard covariates).

These standard covariates do not account for the structure of the tissues and its random geometry. Discriminant analysis is aimed at evaluating the impact of the inclusion of global "morphometric" characteristics computed at the scale of the entire tissues. Moran's and Geary's indices are examples that account for the global structure of the tissues. The new indices $\rho_{\theta}$ are expected to provide finer analyses and indications about the level at which the cell responses interact.

\subsection{Results}

A dataset corresponding to TMAs cores of 31 cases (patients) fixed on a single slide has been utilized 
Table 1. Sensitivities and specificities of linear discriminant analysis (LDA) and cross-validated neural networks (Nnet).

\begin{tabular}{lcc}
\hline & Sensitivity & Specificity \\
\hline LDA & 0.16 & 0.96 \\
Nnet & 0.0 & 1.0 \\
\hline
\end{tabular}

(section 2). The stained scores extracted from digital processing were rescaled and took values between 0 and 255. The scores considered were the cell (Cell.OD), the nucleus (Nucleus.OD) and cytoplasm (Cyto.OD) average optical densities of the biomarker measured locally. Among all available scores, these scores were chosen as the most representative for this specific dataset.

The discriminant power of tests were evaluated thanks to the measures of sensitivity (probability for a test result to be positive when the disease is present, i.e. true positive rate) and specificity (probability for a test result to be negative when the disease is not present, i.e. true negative rate). The data set contains 25 outcomes of disease (carcinoma recurrence) and 6 outcomes of non-disease.

First, linear discriminant analysis and logistic regression were performed using a pool of core measurements. The pool contained six measures corresponding to the mean and standard deviation of the three scores, Cell.OD, Nucleus.OD and Cyto.OD, computed for each case.

Logistic regression was performed using $R$ feedforward neural networks library nnet. The number of hidden units in feedforward neural networks were determined using the jacknife method of cross-validation. The results are displayed in table 1. These results indicate low sensitivity and high specificity. The poor performances may be due to the fact that the three first covariates (means of OD) are strongly correlated (cor $>0.95$ ).

Tables 2-4 summarize the performance of the six measures (mean and sd of Cell.OD, Nucleus.OD and Cyto.OD) considered separately in discriminating the disease. The performances of Moran and Geary's index $M_{\theta}$ and $\rho_{\theta}$ obtained from the ROC curve analysis are displayed as well. Regarding $M_{\theta}$ and $\rho_{\theta}$, the value $\theta=127$ led to the optimal classification performances. The sensitivities range between 0.67 and 0.83 . Because the data set contained only 6 non-disease cases,

Table 2. Score $=$ Cell average optical density (Cell.OD)

\begin{tabular}{lcc}
\hline & Sensitivity & Specificity \\
\hline M.Cell.OD & 0.83 & 0.48 \\
SD.Cell.OD & 0.83 & 0.64 \\
$\rho_{M}$ & 0.67 & 0.72 \\
$\rho_{G}$ & 0.67 & 0.76 \\
$\rho_{\theta}$ & 0.83 & 0.76 \\
\hline
\end{tabular}

Sensitivities and specificities from ROC curve analyses for mean scores (M.Cell.OD), standard deviation of scores (SD.Cell.OD), Moran's $\left(\rho_{\mathrm{M}}\right)$, Geary's $\left(\rho_{\mathrm{G}}\right), \rho_{\theta}(\theta=127)$.
Table 3. $\quad$ Score $=$ Nucleus average optical density (Nucleus.OD).

\begin{tabular}{lcc}
\hline & Sensitivity & Specificity \\
\hline M.Nucleus.OD & 0.83 & 0.44 \\
SD.Nucleus.OD & 0.83 & 0.64 \\
$\rho_{M}$ & 0.67 & 0.72 \\
$\rho_{G}$ & 0.67 & 0.72 \\
$\rho_{\theta}$ & 0.83 & 0.76 \\
\hline
\end{tabular}

Sensitivities and specificities of ROC curve analyses for mean scores (M.Nucleus.OD), standard deviation of scores (SD.Nucleus.OD), Moran's $\left(\rho_{\mathrm{M}}\right)$, Geary's $\left(\rho_{\mathrm{G}}\right), \rho_{\theta}(\theta=127)$.

the difference $0.16=1 / 6$ corresponds to the misclassification of a single case. This variation in sensitivity should therefore be considered with caution. Specificities range from 0.48 (12/25 for the mean of Cell.OD) to 0.76 $(19 / 25)$ for spatial correlation parameter. Therefore considering spatial correlation indices leads to a significant gain in specificity.

\section{Discussion}

Scientific progress in the understanding of tissues, their function, disease and biodynamics critically depend on the precision and quality of information available. Tissue specimens have been the primary source for medical diagnosis since the origin of microscopy. Despite fundamental progress in this area, the prevailing method for analysing tissues today remains visual inspection, and relevant quantification of TMAs core images is still missing.

Many morphological parameters may be used in the grading of malignancy of cancerous tissue. For example, the shape of the cells in the tissue is an important feature. In addition, the spatial conformation of cells in which genes may be over- or under-expressed seems critical [19]. At present these parameters are usually estimated subjectively by the pathologists or described by simple ratios. Idealised mathematical models of tissues can provide further understanding of tissue contents and improve the accuracy of discrimination between samples or experimental groups. The crucial issues addressed in this study were the following: Are standard covariates such as mean and variance of stained scores able to provide discrimination between diseased and non-diseased cases?

Table 4. Score $=$ Cytoplasm average optical density (Cyto.OD).

\begin{tabular}{lcc}
\hline & Sensitivity & Specificity \\
\hline MSS & 0.83 & 0.48 \\
DSS & 0.83 & 0.64 \\
$\rho_{M}$ & 0.67 & 0.72 \\
$\rho_{G}$ & 0.67 & 0.76 \\
$\rho_{\theta}$ & 0.83 & 0.76 \\
\hline
\end{tabular}

Sensitivities and specificities of ROC curve analyses for mean scores (M.Cyto.OD), standard deviation of scores (SD.Cyto.OD), Moran's $\left(\rho_{\mathrm{M}}\right)$, Geary's $\left(\rho_{\mathrm{G}}\right), \rho_{\theta} ;(\theta=127)$. 
Is finer level analysis necessary? Do the thresholded indices $\rho_{\theta}$ provide additional insights in the disease prognosis?

The results presented in the fourth section proved that including specific tissue structure parameters such as Moran and Geary or thresholded indices led to increased classification performances. Nevertheless, these results also raised a series of issues. A first issue concerns the biomarker DARPP-32 itself. The strong correlation of standard covariates computed at the levels of cells, nuclei and cytoplasm indicates that DARPP-32 has no preferential attachment to one of these biological entities, and further experimental evidence is obviously necessary to assess the potential of DARPP-32 as a therapeutical target.

A second issue concerns the choice of a threshold $\theta$ or equivalently the choice of a subset $B_{\theta}$ for use in the computation. In our analysis, the threshold was optimized in order to produce the best classification performances. The resulting parameter was found to be close to the median score. However, choosing a subset $B_{\theta}$ of the form $B_{\theta}=$ $\left\{i \in B, X_{i}>\theta\right\}$ may not always be an optimal strategy. Consider for instance a biomarker which has different expressions in cytoplasm and nucleus according to the pathologic state of the tissue. In this situation, $B_{\theta}$ could be taken as the subset of cells with significant differential expression. Because the distribution of covariates (e.g. Nucleus.OD and Cyto.OD) is hardly Gaussian, additional normalisation steps would be necessary in order to eliminate biases which may come from spurious sources. MA-normalised variables [20] are plotted in figures 2 and 3 . In this figure $M$ represents the intensity log-ratio $M=\log$ Nucleus.OD/Cyto.OD and $A$ is the arithmetic average $A=\log \sqrt{\text { Nucleus.ODCyto.OD. }}$

Statistics are often used to test null hypotheses. However, testing hypotheses of elementary relationships



Figure 2. Plot of correlated stained scores Nucleus.OD vs. Cyto.OD for a typical MicroArray core before normalization (6000 cells).

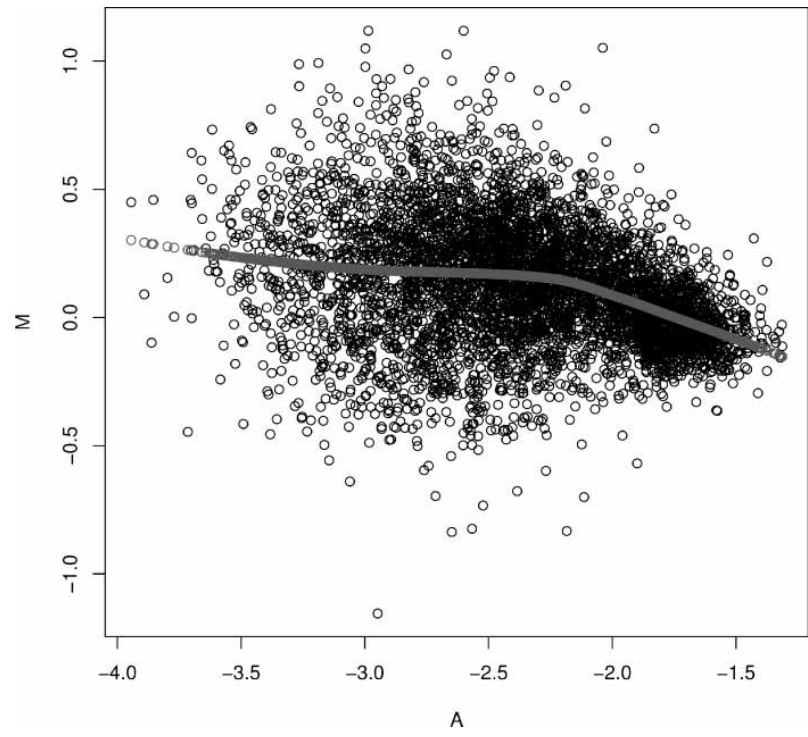

Figure 3. Plot of correlated stained scores Nucleus.OD vs. Cyto.OD for a typical MicroArray core after MA-normalization. A robust regression curve (loess) is also displayed and suggest a systematic way of suppressing biases.

between genetic expression, and tissue structures coupling spatial architecture and gene expression, would rely on null models of interactions at the cellular level. Such models could integrate measures of organisation or disorder using basic ideas of statistical mechanics. Changes in gene expression are responsible for and correlate with changes in tissue structural feature expression. Just as genetic expression results in unique macroscopic differences between individuals, it is likely that gene expression regulates unique microstructural differences between tissues from different individuals. Our next step in modeling tissues would rely on a GibbsBoltzmann formalism. Computationally tractable GibbsBoltzmann models of interactions between cells would involve tissue geometry through simplified models of architecture such as the Voronoi tesselation presented in this article [21]. Because these models include measures of organisation and disorder at the scale of the tissue, estimating interaction parameters could provide more direct understanding of the architecture than correlation parameters. Thus changes in tissue histology and gene expressions could be related to phase transitions in the model. The strength of these different methodologies is that their combination could enable statistically wellfounded quantification of the potential of genes expression for correlation with phenotypical responses. Such combined analyses can be applied to conditions where gene expression and tissue histology are integral to the pathophysiology of disease.

\section{Acknowledgements}

The authors are deeply indebt to E. Bertin for this overall participation in the TMA analysis project in Grenoble. 


\section{References}

[1] Kononen, J., Bubendorf, L., Kallioniemi, A., Barlund, M., Schraml, P., Leighton, S., Torhorst, J., Mihatsch, M.J., Sauter, G. and Kallioniemi, O.P., 1998, Tissue microarray for high-throughput molecular profiling of tumor specimens. Natural Medicine, 4, 844-847.

[2] Nocito, A., Bubendorf, N.A., Maria Tinner, E., Suess, K., Wagner, U., Forster, T., Kononen, J., Fijan, A., Bruderer, J., Schmid, U., Ackermann, D., Maurer, R., Alund, G., Knonagel, H., Rist, M., Anabitarte, M., Hering, F., Hardmeier, T., Schoenberger, A.J., Flury, R., Jager, P., Luc Fehr, J., Schraml, P., Moch, H., Mihatsch, M.J., Gasser, T. and Sauter, G., 2001, Microarrays of bladder cancer tissue are highly representative of proliferative index and histological grade. Journal of Pathology, 194, 349-357.

[3] Stoyan, D., Kendall, W. and Mecke, J., 1995, Stochastic Geometry and its Applications (Chichester: John Wiley and Sons).

[4] Moran, P.A.P., 1950, A test for serial independence of residuals. Biometrika, 37, 178-181.

[5] Geary, R.C., 1954, The contiguity ratio and statistical mapping. The Incorpored Statistician, 5, 115-145.

[6] Møller, J., 1999, Topics in Voronoi and Johnson-Mehl tessellations. In: O.E. Barndorff-Nielsen, W.S. Kendall and M.N.M. Van Lieshout (Eds.) Monographs on Statistics and Applied Probability (Boca Raton: Chapman and Hall/CRC), pp. 173-198.

[7] Bettencourt, M.C., Bauer, J.J., Sesterhenn, I.A., Mosto, F.K., McLeod, D.G. and Moul, J.W., 1996, Ki- 67 expression is a prognostic marker of prostate cancer recurrence after radical prostatectomy. Journal of Urology, 156, 1064-1068.

[8] Chung, G.G., Provost, E., Kielhorn, E.P., Charette, L.A., Smith, B.L. and Rimm, D.L., 2001, Tissue microarray analysis of betacatenin in colorectal cancer shows nuclear phospho-beta-catenin is associated with a better prognosis. Clinical Cancer Research, 7, 4013-4020.

[9] Maass, N., Hojo, T., Zhang, M., Sager, R., Jonat, W. and Nagasaki, K., 2000, Maspin-a novel protease inhibitor with tumorsuppressing activity in breast cancer. Acta Oncologica, 39, 931-934.

[10] Zhang, W. and Zhang, M., 2002, Tissue microarray analysis of maspin expression and its reverse correlation with mutant p53 in various tumors. International Journal of Oncology, 20, $1145-1150$

[11] Gehrig, P.A., Van Le, L., Olatidoye, B. and Geradts, J., 2000, Estrogen receptor status determined by immunohistochemistry as a predictor of the recurrence of stage 1 endometrial carcimona. Cancer, 86, 2083-2102.

[12] Liu, X., Minin, V., Huang, Y., Seligson, D.B. and Horvath, S., 2004, Statistical methods for analysing tissue microarray data. UCLA, Preprint.

[13] Beckler, A., Moskaluk, C.A., Zaika, A., Hampton, G.M., Powell, S.M., Frierson, Jr. H.F. and El-Rifai, W., 2003, Overexpression of the 32-kilodalton dopamine and cyclic adenosine $3^{\prime}, 5^{\prime}$-monophosphate-regulated phosphoprotein in common adenocarcinomas. Cancer, 98(7), 1547-1551.

[14] Liu, C.L., Prapong, W., Natkunam, Y., Alizadeh, A., Montgomery, K., Blake Gilks, C. and Van de Rijn, M., 2002, Software tools for high-throughput analysis and archiving of immunohistochemistry staining data obtained with tissue microarrays. American Journal of Pathology, 161, 1557-1565.

[15] Honda, H., 1978, Description of cellular patterns by Dirichlet domains: The two dimensional case. Journal of Theoretical Biology, 72, 523-543.

[16] Besag, J., 1975, Statistical analysis of non-lattice data. The Statistician, 24, 179-195.

[17] Apanasovich, T.V., Sheather, S., Lupton, J.R., Popovic, N., Turner, N.D., Chapkin, R.S., Braby, L.A. and Caroll, R.J., 2003, Testing for spatial correlation in nonstationary binary data, with application to aberrant crypt foci in colon carcinogenesis. Biometrics, 59, 752-761.

[18] Zweig, M.H. and Campbell, G., 1993, Receiver-operating characteristic (ROC) plots: a fundamental evaluation tool in clinical medicine. Clinical Chemistry, 39, 561-577.

[19] Bissell, M.J., Weaver, V.M., Lelievre, S.A., Wang, F., Petersen, O.W. and Schmeichel, K.L., 1999, Tissue structure, nuclear organization, and gene expression in normal and malignant breast. Cancer Research, 59(7), 57-1763.

[20] Dudoit, S., Yang, Y.H., Callow, M.J. and Speed, T.P., 2002, Statistical methods for identifying differentially expressed genes in replicated cDNA microarray experiments. Statistica Sinica, 12, 111-139.

[21] Bertin, E., Billiot, J.M. and Drouilhet, R., 1999, Spatial Delaunay Gibbs point processes. Stochastic Models, 15(2), 181-199. 


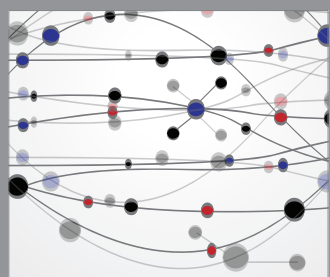

The Scientific World Journal
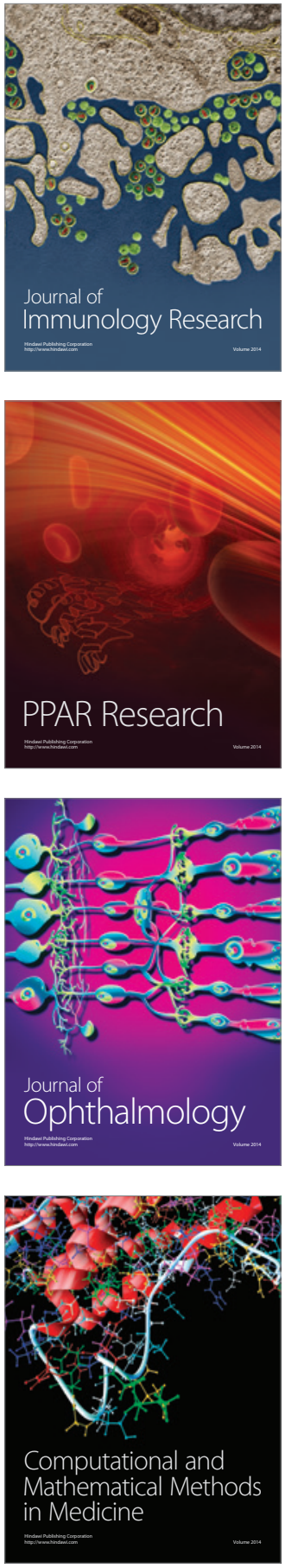

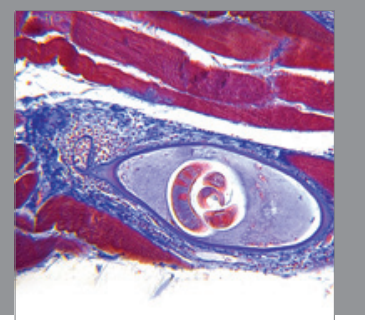

Gastroenterology

Research and Practice
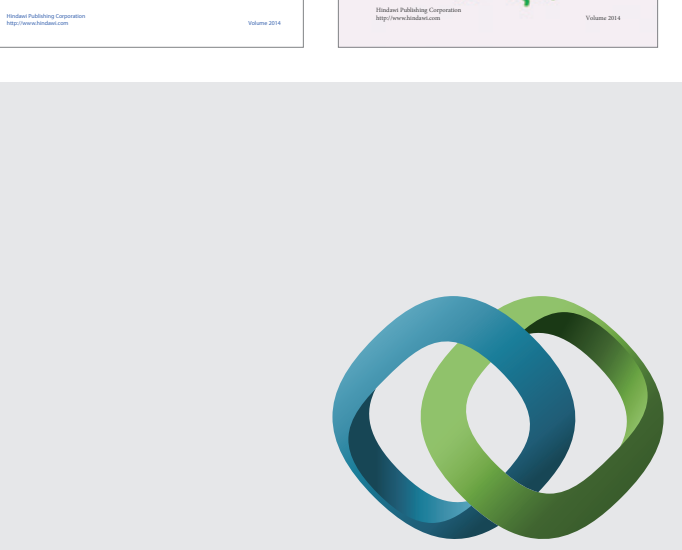

\section{Hindawi}

Submit your manuscripts at

http://www.hindawi.com
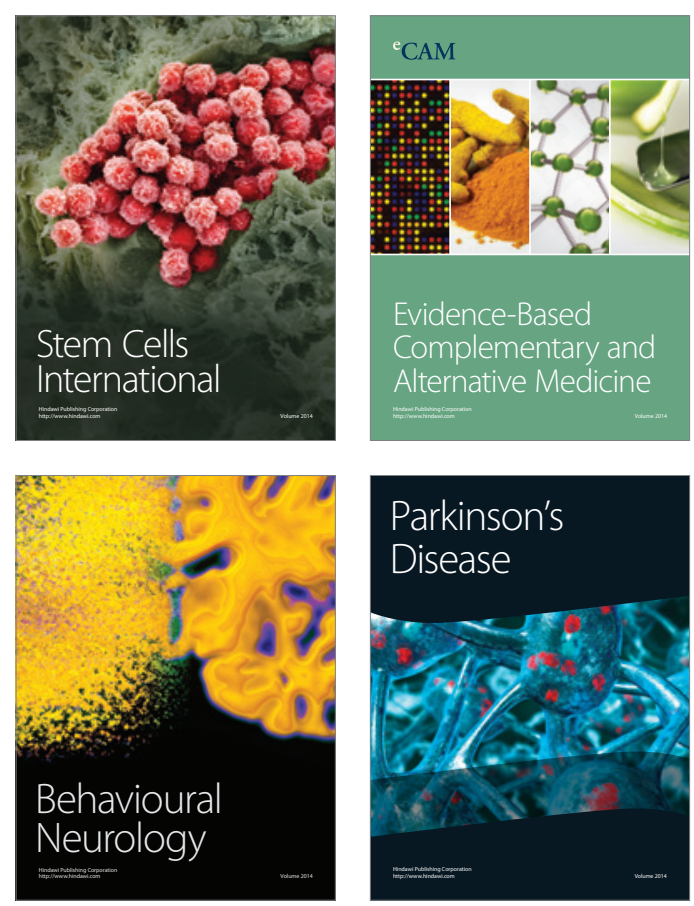

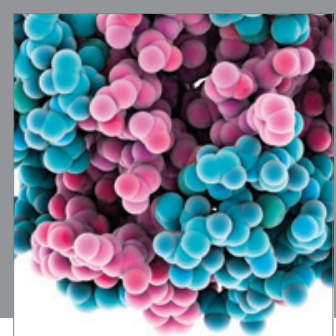

Journal of
Diabetes Research

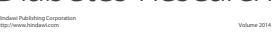

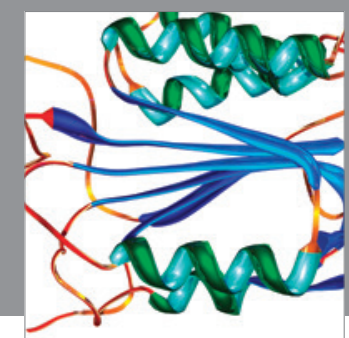

Disease Markers
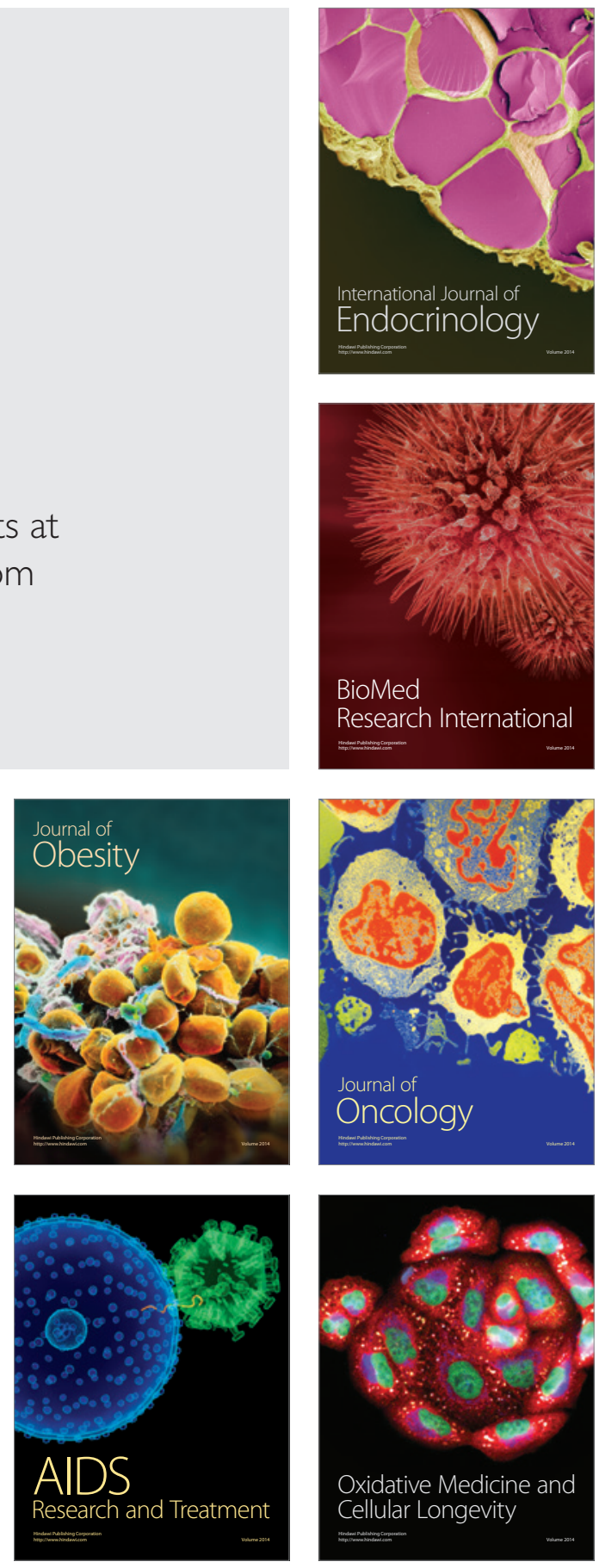